\title{
Electron Spin-Lattice Relaxation in Polymers and Crystals Related to Disorder and Structure Defects
}

\author{
S.K. HoffmanN*, W. HLlczer, T. RAdCZYK \\ Institute of Molecular Physics, Polish Academy of Sciences \\ M. Smoluchowskiego 17, 60-179 Poznań, Poland
}

AND I. POLUS

A. Cieszkowski Agriculture University, Faculty of Wood Technology Department of Chemistry, Wojska Polskiego 75, 60-625 Poznań, Poland

(Received August 16, 2002; revised version November 21, 2002; in final form December 18, 2002)

Temperature dependences $(4-300 \mathrm{~K})$ of the electron spin-lattice relaxation have been determined by electron spin echo technique for free radicals in two polymeric systems: phenol-formaldehyde resin and polyhydrazodisulphide. The dependences are described in terms of dynamics specific for amorphous systems involving two-level tunnelling states at low temperatures, exchange-coupled clusters of paramagnetic centres and local oscillators. Some universal temperature behaviour of the spin-lattice relaxation of amorphous systems is suggested, with a strong increase in relaxation rate with temperature at low temperatures and much weaker increase above $50 \mathrm{~K}$ with characteristic linear temperature dependence in a broad temperature range and $\operatorname{cosech}(\Delta / k T)$-type behaviour. It is also shown that the amorphous-type behaviour appears in low temperatures relaxation studies of single crystals but it is due to a non-uniform distribution of doped paramagnetic ions. Such behaviour we have found in Tutton salt crystals doped with $\mathrm{Cu}^{2+}$, as well as for free radical centres produced by ionising irradiation in $\left(\mathrm{NH}_{4}\right)_{3} \mathrm{H}\left(\mathrm{SeO}_{4}\right)_{2}$ and $\mathrm{Li}\left(\mathrm{N}_{2} \mathrm{H}_{5}\right) \mathrm{SO}_{4}$ single crystals, where extended phonons are suppressed around radiation damage centres suggesting a local amorphisation of the crystal structure.

PACS numbers: $61.41 .+\mathrm{e}, 61.80 .-\mathrm{x}, 76.30 .-\mathrm{V}$

*corresponding author; e-mail: skh@ifmpan.poznan.pl 


\section{Introduction}

Amorphous materials like glasses, polymers, biopolymers, and disordered solids exhibit physical properties strikingly different from those of crystalline solids. There is no long-range order in such materials and their properties are determined by disorder and by global and local metastability [1]. Moreover, a phonon concept has restricted validity and a new type of local and lattice dynamics appears. The energy excitations appearing in amorphous solids are: tunnelling two-level systems, soft local oscillators, localized phonons and boson peaks.

At low temperatures amorphous solids exhibit a variety of surprising properties and anomalous thermal, acoustic and optical behaviour. Phonons cannot account for this kind of behaviour; instead localized low-energy excitations are needed. Their density of states have two peculiarities as compared to the Debye-type density: below of about $1 \mathrm{~K}$ the density of states is constant then increases rapidly and in temperature range of 3-15 K it excesses 2-6 times the Debye density.

The first anomaly is attributed to the existence of tunnelling two-level systems (TLS) [2]. TLS can be pictured as a shallow double well potential of which each well represents a distinct configuration of the amorphous solid. Even at low temperatures the transitions between the wells appear due to the tunnelling or phonon-assisted tunnelling. The solid is thought to consist of a large ensemble of the TLS's with a broad distribution of energy separation between the wells and tunnel parameters, causing a broad distribution of the fluctuation rates.

A rapid increase in the excitation density of states on heating with $\rho(E) \propto E^{4}$ is explained as due to local oscillators being low frequency collective molecular excitations localized within 1-2 nm [3]. The phonons in amorphous systems are localized, in Anderson's sense, and are scattered by the local oscillators. When a number of the oscillators increases with temperature the free path of phonons decreases and at some temperature the free path becomes comparable with the phonon wavelength and the phonon concept losses the sense [4]. Above this temperature there are no phonons in a system. Interactions between local oscillators increase with temperature producing their localization and then the oscillators conduct the heat in a system.

Another type of excitation in disordered solids is so called boson peak. It contains a broad spectrum of low frequency harmonic excitations obeying the Bose-Einstein statistics [2,4]. This peak is especially pronounced in strong glasses (glass formers). The peak excesses the calculated Debye density of states and is located in a range of $10-100 \mathrm{~cm}^{-1}$. Its origin is still not clear but it was shown that in vitreous silica the boson peak is due to the rocking motions (librations) of the $\mathrm{SiO}_{4}$-tetrahedra [4].

Electron spin-lattice relaxation in amorphous systems is governed by their specific dynamics and indicates a strict difference as compared to the ionic crystals. There are two specific features characteristic of amorphous materials: (1) The relaxation rate $1 / T_{1}$ at low temperatures (below $20 \mathrm{~K}$ ) can be a few order of 
magnitudes faster than that in single crystals and exhibits a linear increase with temperature [5], whereas in crystals the $1 / T_{1} \propto T^{9}$ (for Kramers transitions) and is of order of $10^{-2} \mathrm{~s}^{-1}$ at helium temperatures [6]; (2) The relaxation rate much slowly increases with temperature (in most cases as $1 / T_{1} \propto \operatorname{cosech}(\Delta / k T)$ ), typically $3-5$ decades in a temperature range of $4-300 \mathrm{~K}$, whereas very strong increases (8-10 decades) are observed for paramagnetic ions in single crystals due to the two-phonon Raman relaxation processes [7].

There are not many papers dealing with electron spin relaxation in amorphous materials. They are summarized by Misra [8] and are related mainly to inorganic glassy materials. In this paper we have collected the published data for polymers and added our new results for two polymeric systems. We review shortly the existing relaxation theories and analyse experimental data in terms of these theories. Moreover, we have collected our published results for single crystals, which clearly show effects characteristic of amorphous materials.

\section{Electron spin-lattice relaxation mechanisms and temperature dependence of the relaxation rate}

Both the TLS and local oscillators can deliver mechanisms of the spin-lattice relaxation. Also well-defined local vibrational modes, like reorientations or librations of molecular groups, can be dominant relaxation mechanism at some temperature range. Moreover a non-uniform distribution of paramagnetic centres can significantly influence the relaxation.

Relaxation due to the TLS dynamics can be generally described assuming that tunnel reorientations between potential wells produce simultaneously the spin flips. Assuming the thermal equilibrium, the relaxation rate is [9]

$$
\frac{1}{\tau_{\mathrm{c}}}=A \delta^{2} E \operatorname{coth}\left(\frac{E}{2 k T}\right)+\frac{1}{\tau_{0}} \cosh \left(\frac{\delta}{2 k T}\right) \exp \left(-\frac{V}{k T}\right),
$$

where parameters describing the double-well asymmetric potential are defined in Fig. 1. The first term results from phonon-assisted tunnelling ( $A$ is a matrix ele-

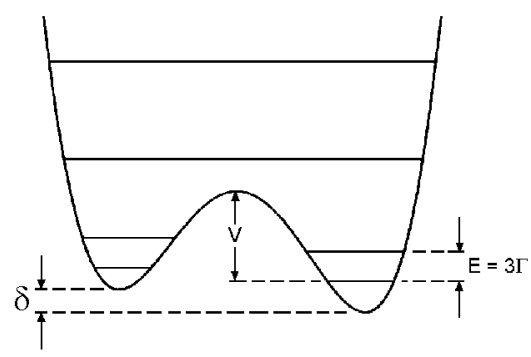

Fig. 1. Asymmetric double potential well model of TLS. 
ment of the phonon-tunnelling coupling), while the second term describes classical over-barrier jumps with $\tau_{0}$ being the relaxation time of the TLS states. If the $\tau_{\mathrm{c}}$ is the correlation time of an exponential correlation function the spin-lattice relaxation rate can be calculated from the Fermi golden rule as $[8,10]$

$$
\frac{1}{T_{1}}=\frac{2}{\hbar^{2}}\left|\left\langle\sigma_{1}\left|H_{\mathrm{T}-\mathrm{S}}\right| \sigma_{2}\right\rangle\right|^{2} \int_{0}^{\delta_{m}} \int_{0}^{V_{m}} \frac{f(\delta, V)}{\cosh ^{2}(\delta / 2 k T)} \frac{\tau_{\mathrm{c}}}{1+\omega^{2} \tau_{\mathrm{c}}^{2}} \mathrm{~d} \delta \mathrm{d} V
$$

where $f(\delta, V)$ is a distribution function with maximal parameters: $\delta_{m}$ for energy difference, and $V_{m}$ for the barrier height.

A specific relaxation mechanism has been proposed for glassy state, where it was assumed that TLS dynamics modulates the dipolar nuclear hyperfine coupling between unpaired electron and magnetic nucleus of TLS. The relaxation rate for this mechanism can be calculated as $[8,11]$

$$
\frac{1}{T_{1}}=A_{\mathrm{TLS}} \frac{1}{\omega^{2}} \int_{0}^{\infty} E \operatorname{cosech}\left(\frac{2 E}{k T}\right) f(E) \mathrm{d} E,
$$

where $E=3 \Gamma$ is tunnelling splitting and $f(E)$ is its distribution function. A detailed consideration of Eqs. (2) and (3) shows that temperature dependence of the relaxation rate can be approximated by quadratic dependence $1 / T_{1} \propto T^{2}$ at low temperatures $(k T<2 E)$ and by linear dependence at high temperatures $(k T>2 E)$.

In solids with defects the quasi-local or local vibrations can appear with a large amplitude and anharmonicity in the neighbourhood of a point defect. Such local oscillators contribute significantly to the electron spin relaxation. Raman-type relaxation process via local oscillator of frequency $\omega_{\mathrm{L}}$ leads to the temperature dependence of the relaxation rate $[12,13]$ :

$$
\frac{1}{T_{1}}=A_{\text {Local }} \operatorname{cosech}^{2}\left(\frac{\hbar \omega_{\mathrm{L}}}{k T}\right) .
$$

For $\hbar \omega_{\mathrm{L}}>k T$ this dependence becomes quadratic $1 / T_{1} \propto T^{2}$ which is characteristic of high temperature Raman process.

Paramagnetic centres doped to or generated in an amorphous solid are often non-uniformly distributed thus the exchange or dipolar coupled pairs, triads or larger clusters of paramagnetic centres can exist. The spin-lattice relaxation in clusters is much faster than that for individual centres since a modulation of the singlet-triplet splitting $\Delta_{\mathrm{ST}}$ by the local dynamics is a very effective relaxation mechanism. It was calculated [8] that this mechanism produces temperature dependence of the pair relaxation rate, which can be approximated by a quadratic term $\left(\propto T^{2}\right)$ below of about $10 \mathrm{~K}$ and linear term dominating at intermediate temperatures. For relatively large concentration of paramagnetic centres having uniform distribution the cross-relaxation between exchange coupled pairs and individual spins becomes important relaxation mechanism at higher temperatures. When the spin-lattice relaxation dominates over the cross-relaxation the expected relaxation rate is $[8,14]$ 


$$
\frac{1}{T_{1}} \propto c^{2} \frac{1}{1+\exp \left(\Delta_{\mathrm{ST}} / k T\right)}
$$

where $c$ is the spin concentration. When the cross-relaxation dominates [8, 15]

$$
\frac{1}{T_{1}} \propto c \Delta_{\mathrm{ST}}^{3} \frac{1}{\sinh \left(\Delta_{\mathrm{ST}} / k T\right)}=c \Delta_{\mathrm{ST}} \operatorname{cosech}\left(\frac{\Delta_{\mathrm{ST}}}{k T}\right) .
$$

In high temperature range when $\Delta_{\mathrm{ST}}<k T \mathrm{Eq}$. (5) predicts that the relaxation rate is temperature independent whereas Eq. (6) predicts a linear temperature dependence.

One can expect that a distribution of the parameters describing the relaxation mechanisms will result in a distribution of the relaxation rates. In such a case the experimentally measured recovery of the magnetization after an excitation should be described by a stretched exponential function

$$
M_{z}=M_{0}\left[1-\exp \left(\frac{t}{T_{1}}\right)^{\beta}\right]
$$

Very often such behaviour is indeed observed, although in many cases despite the disorder in a system the magnetization recovery is surprisingly single exponential with $\beta=1$. Such situation has been found in highly inhomogeneous coal samples [16]. It seems that it can appear when there exists a very finite range of the distribution or when the local dynamics is fast enough to average out disorder in the time scale of the spin-lattice relaxation.

\section{Experimental}

We have measured the electron spin-lattice relaxation in two polymeric materials, where free radicals occur spontaneously as residuals of polymerisation reactions. The spin-lattice relaxation was measured by electron spin echo technique using a Bruker ESP 380E spectrometer equipped with Oxford CF935 cryostat. Relaxation time $T_{1}$ was determined by the saturation recovery method after saturation by a short microwave pulse $(16-48 \mathrm{~ns})$. The short pulse of $t_{\mathrm{p}}=16 \mathrm{~ns}$ duration have the spectral width $\nu_{\text {band }}=1.207 / t_{\mathrm{p}}=75 \mathrm{MHz}$ (in magnetic field scale $\left.B_{\text {band }}(\mathrm{mT})=0.0714 \nu_{\text {band }}(\mathrm{MHz}) / g=2.68 \mathrm{mT}\right)$ and is able to saturate the whole EPR spectra in the studied compounds. The recovery of the magnetization was monitored by the amplitude of electron spin echo (ESE) signal of the Hahn-type generated by two 16 ns pulses. Spectral diffusion and instantaneous diffusion effects were negligible.

\section{Results}

\subsection{Phenol-formaldehyde resin}

This is a thermosetting polymer used as an adhesive in the plywood industry. When the curing reaction is stopped on the first stages of the cross-linking, the dried material prepared for a shelf-storage displays the weak EPR signal. We have 

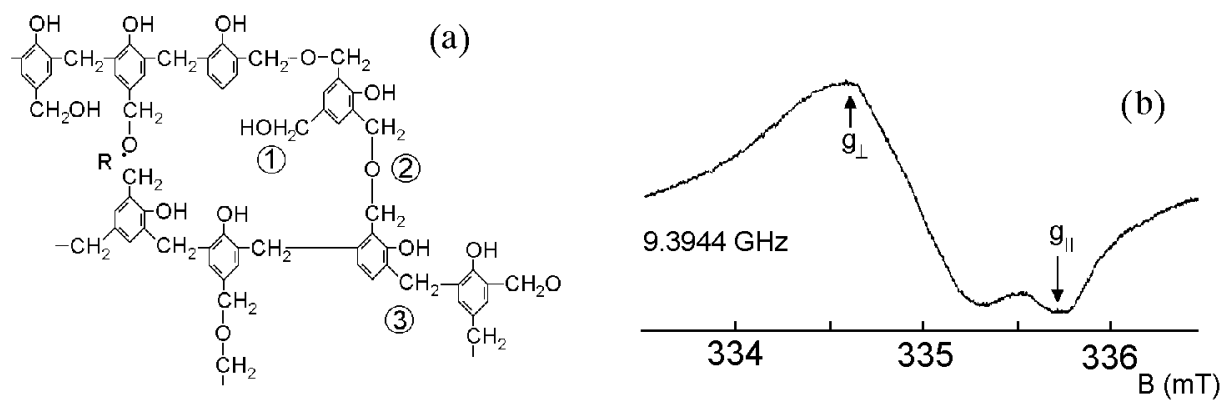

Fig. 2. (a) Molecular structure of partially cross-linked phenyl-formaldehyde resin with free radical (marked by the dot) localized on the oxygen atom; 1 - methylol group $-\mathrm{CH}_{2} \mathrm{OH} ; 2$ - dimethylene ether bridge $-\mathrm{CH}_{2} \mathrm{OHCH}{ }_{2}-; 3-$ methylene bridge $-\mathrm{CH}_{2}-$. (b) X-band EPR spectrum at $282 \mathrm{~K}$ with $g_{\|}=1.9994$ and $g_{\perp}=2.0058$.

identified the signal as due to the broken dimethylene ether linkage (Fig. 2a) [17]. The signal shows axial $g$-tensor anisotropy with $g_{\|}=1.9994(6), g_{\perp}=2.0058(9)$ (Fig. $2 \mathrm{~b}$ ) and $10^{16}-10^{17}$ radicals/gram. The concentration of radicals increases slowly with time up to 100 days after polymerisation. The electron spin relaxation measurements on the fresh samples we already published [17]. ESE signal in aging samples was detectable in the whole temperature range of $4.2-300 \mathrm{~K}$ indicating inhomogeneous EPR line broadening due to weak dipolar coupling with distant hydrogen nuclei. The magnetization recovery after pulse excitation was
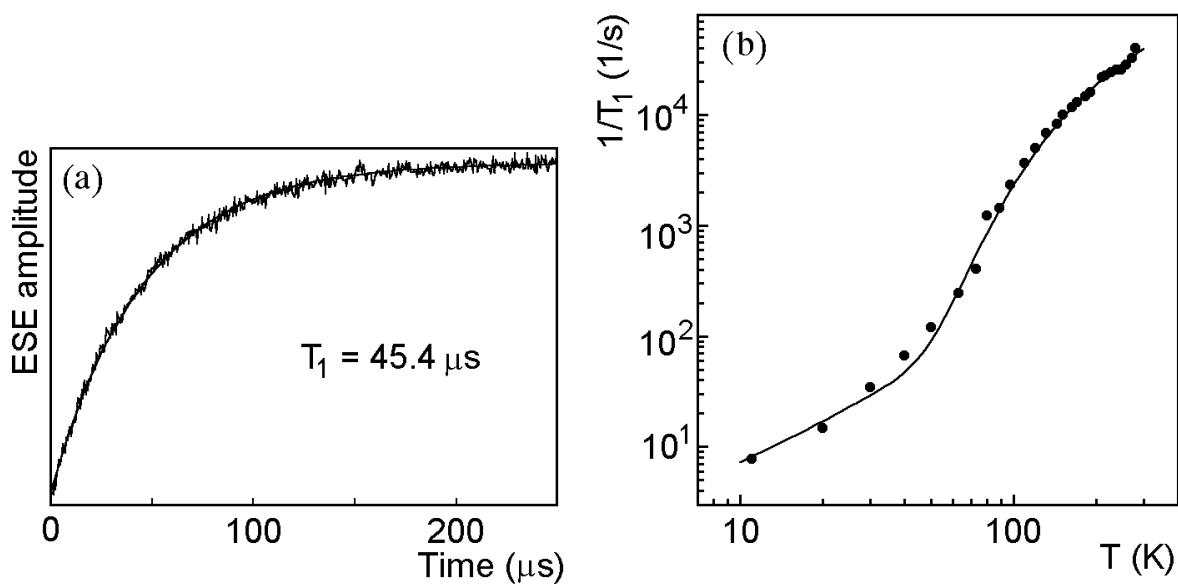

Fig. 3. (a) Recovery of the magnetization of the radical system in phenyl-formaldehyde resin after pulse saturation at $210 \mathrm{~K}$ with solid line being the best fit with single exponential function. (b) Temperature dependence of the relaxation rate $1 / T_{1}$ in $\log -\log$ scale for radicals in phenyl-formaldehyde resin with the solid line being the best fit to Eq. (8). 
single exponential in the whole temperature range as is shown in Fig. 3a. The relaxation rate $1 / T_{1}$ increases in about 5 orders of magnitude in this temperature range (Fig. 3b) and is described by the equation

$$
\frac{1}{T_{1}}=A_{1} T+B \operatorname{cosech}\left(\frac{\Delta}{k T}\right)
$$

with parameters: $A_{1}=0.30 \mathrm{~K}^{-1} \mathrm{~s}^{-1}, B=2.78 \times 10^{4} \mathrm{~s}^{-1}$ and $\Delta=390 \mathrm{~K}=$ $201 \mathrm{~cm}^{-1}=2.35 \mathrm{~kJ} / \mathrm{mol}$. The linear term dominates up to $50 \mathrm{~K}$. At higher temperatures excitations with energy $\Delta$ are dominating relaxation mechanism. This is a typical behaviour of the relaxation in amorphous and disordered solids. The presented results show that the long lasting aging of the samples, producing an increase in radical concentration, have a negligible effect on the electron spin relaxation dominated by local dynamics.

\subsection{Poly-hydrazodisulphides with $N$-methyl-2pyrrolidone end chains}

After polycondensation reaction of parent substances the solid oligomer samples (Fig. 4a) display a strong EPR signal, which consists of a single symmetrical nearly Lorentzian line (Fig. 4b). Electron spin echo envelope modulation (ESEEM) spectrum has allowed us to show that the signal is produced by a free radical with unpaired electron located on the carbon atom of the end chain pyrrolidone molecule [18]. The ESE signal was detected in the whole temperature range of $4.2-300 \mathrm{~K}$ but magnetization recovery after saturation was not a single exponential but well fitted with stretched exponential function of Eq. (7). The $\beta$-coefficient characterizing the width of the $T_{1}$-time distribution is $\beta=0.58$ at $10 \mathrm{~K}$ and linearly increases with temperature up to $\beta=0.92$ at $300 \mathrm{~K}$. This indicates that an increase in molecular dynamics with temperature is much faster than the increase in the relaxation rate and the dynamics tends to average the disorder effects in the relaxation. In fact the relaxation rate is very weakly temperature dependent

(a)
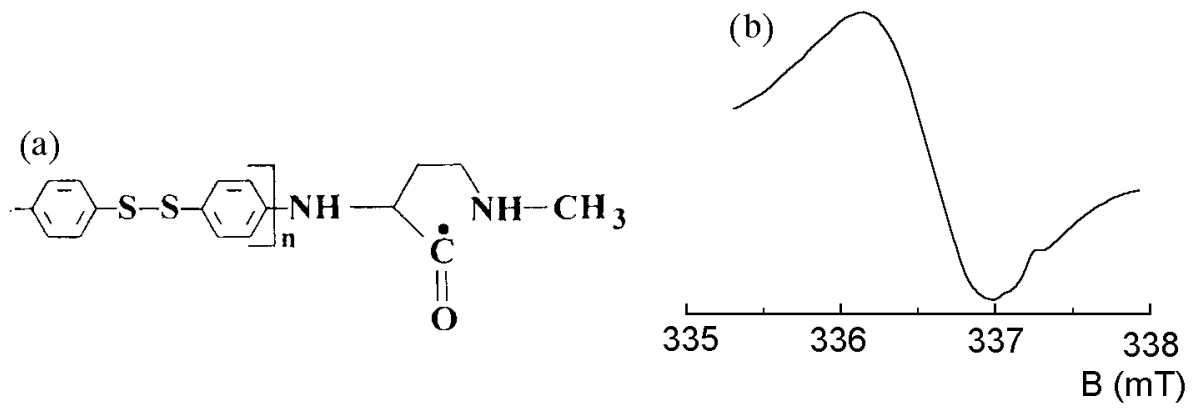

Fig. 4. (a) Polyhydrazodisulphide oligomer with free radical (marked by the dot) located on the carbon atom of the N-methyl-2-pyrrolidone end chain. (b) X-band $(9.4 \mathrm{GHz}$ ) EPR signal at $295 \mathrm{~K}$ with $g=2.0025$. 


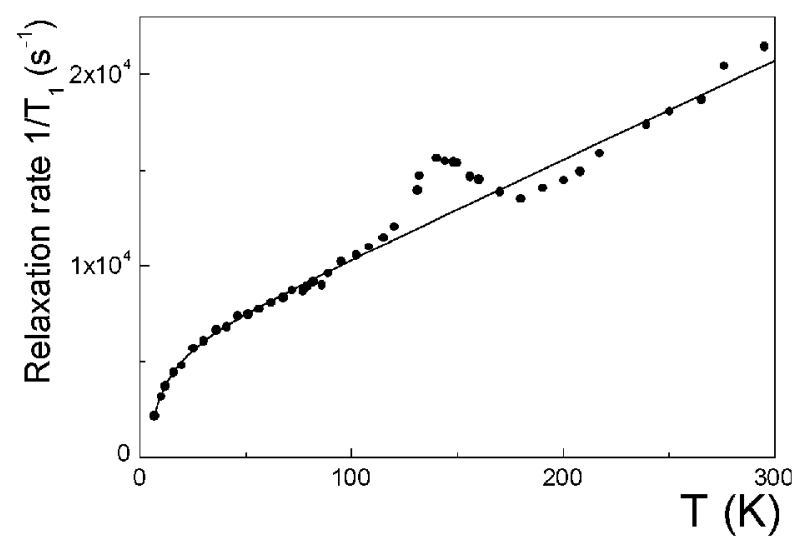

Fig. 5. Temperature dependence of the radical in polyhydrazodisulphide polymer with the solid line being the fit to Eq. (9) (see the text).

as is shown in Fig. 5. After a rapid nonlinear increase below $25 \mathrm{~K}$ the $1 / T_{1}$ is very weakly linearly temperature dependent as described by the equation

$$
\frac{1}{T_{1}}=A_{2} T+C \frac{1}{1+\exp \left(\Delta_{\mathrm{ST}} / k T\right)}
$$

with $A_{2}=51 \mathrm{~K}^{-1} \mathrm{~s}^{-1}, C=11000 \mathrm{~s}^{-1}$, and $\Delta_{\mathrm{ST}}=7.6 \mathrm{~cm}^{-1}=0.09 \mathrm{~kJ} / \mathrm{mol}$. The second term suggests a cross-relaxation to antiferromagnetically exchange coupled pairs. An unusual effect appears around $150 \mathrm{~K}$, where a resonance-type enhancement of the relaxation rate is observed. It can be due to the thermally activated motions of $\mathrm{CH}_{3}$ groups.

\section{Discussion}

Experimental data show that there are not extended phonons in the both polymeric materials. Electron spin-lattice relaxation is not governed by ordinary phonon processes as for paramagnetic ions in crystalline solids but by other mechanisms related to disorder or local dynamics of a polymer. There are two characteristic features of the relaxation in polymers. The first is a rapid increase in the relaxation rate at low temperatures, which can be faster than the $T^{9}$-dependence due to phonon in crystals. This is due to a broad distribution of the low energy excitations like TLS-systems and/or weakly coupled pairs of paramagnetic centres. The second characteristic feature is a relatively slow increase in the relaxation rate at higher temperatures (typically above $50 \mathrm{~K}$ ) due to higher energy excitations like local oscillators or reorienting molecular groups. It is well visible in Fig. 6, where our results are summarized with published data for polymeric systems and compared with data for $\mathrm{Cu}^{2+}$ ions in a single crystal.

Radicals in the both polymeric systems display the linear temperature dependence of $1 / T_{1}$ in a broad temperature range. Such dependence can be produced 


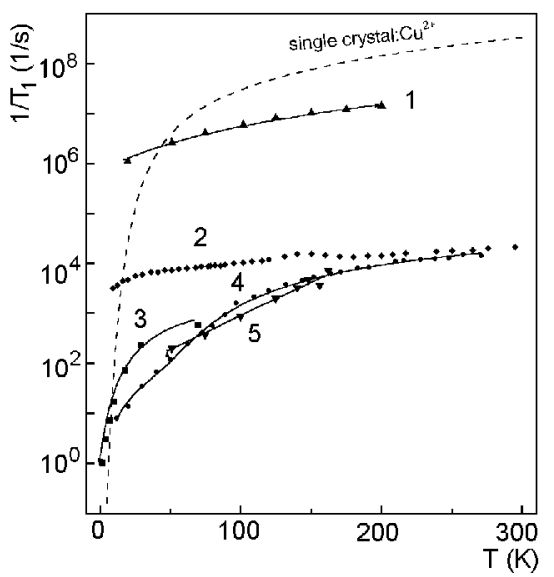

Fig. 6. Temperature dependence of the spin-lattice relaxation rate, in semi-log scale, of free radicals (2-5) and ions (1) in various polymers compared with the rate for $\mathrm{Cu}^{2+}$ in $\left(\mathrm{NH}_{4}\right)_{2} \mathrm{Zn}\left(\mathrm{SO}_{4}\right)_{2} \cdot 6 \mathrm{H}_{2} \mathrm{O}$ single crystal [30] (dashed line): 1 - poly-4-vinylpyridine resin with $\mathrm{Gd}^{3+}[22]$ (triangles); 2 - polyhydrazinodisulphide (this paper) (diamonds); 3 - polyethylene [31] (squares); 4 - phenyl-formaldehyde resin (this paper) (circles); 5 - cation exchanger resin [32] (triangles). Solid lines for 1, 3, and 5 are guides for eyes only.

by various mechanisms as: modulations of the hyperfine coupling by tunnelling TLS at low temperatures, modulations of the singlet-triplet splitting of exchange coupled pairs by local dynamics and/or local oscillators dynamics. In all these cases the linear temperature dependence is a high temperature approximation of equations like Eq. (3) or Eq. (6).

At higher temperatures, above $50 \mathrm{~K}$ as a rule, the experimentally observed temperature dependence is often of the form $1 / T_{1} \propto \operatorname{cosech}(\Delta / k T)$. The function $\operatorname{cosech}(x)=2 /\left(\mathrm{e}^{x}-\mathrm{e}^{-x}\right)$ describes any relaxation process via excitations to the energy level of energy $\Delta$ when the level population cannot be neglected. At low temperatures $\Delta \gg k T$ and the cosech-type relaxation can be well approximated by $1 / T_{1} \propto \exp (-\Delta / k T)$, i.e. typical Orbach process via excited and practically non-populated energy state. At high temperatures $\Delta \ll k T$ and a good approximation is a linear dependence $1 / T_{1} \propto T$. The mechanism of such cosech-type relaxation can be delivered by a local vibrational mode (Murphy's mechanism [19]) like reorientations or tunnelling of a closely located molecular group, by torsional oscillations of a local oscillators, or by a puckering of strained ring systems [20]. Another possible interpretation of the $\Delta$-energy is that this is not energy of a well-defined energy state but energy of an excitation located in the broad boson peak characteristic of disordered materials. In some materials anharmonic local vibrations can contribute to temperature dependence of $1 / T_{1}$ as $\operatorname{cosech}^{2}(\Delta / k T)$ (see Eq. (4)) leading to the $T^{2}$-dependence at high temperatures [13]. Figure 6 
and the above discussion suggest an existence of some universal behaviour of the spin-lattice relaxation of free radicals in polymeric materials despite differences in an effectivity of the relaxation mechanisms at low temperatures or even a background contribution from spectral and instantaneous diffusion visible for the compound 1 with a high concentration of the paramagnetic defects. The universal type behaviour was also suggested for the electron spin-lattice relaxation of paramagnetic ions in amorphous solids [21, 22].

A typical amorphous behaviour can be broken when a polymeric system shows some degree of crystallization, which is well reflected in electron spin-lattice relaxation data. For example the relaxation of $\mathrm{Fe}^{3+}$ ions in reduced $[2 \mathrm{Fe}-2 \mathrm{~S}]$ centre in ferrodoxin [23] is described by the equation

$$
\frac{1}{T_{1}}=0.874 T^{2}+4.4 \times 10^{10} \exp \left(-\frac{348}{T}\right)+0.00341 T^{5.7},
$$

where the first term is characteristic of TLS dynamics and the second describes relaxation due to local dynamics. These two terms give dominant contributions below 20 K. For higher temperatures the last term describing two-phonon Raman processes (written in the power-law form) dominates, producing strong acceleration of the relaxation with temperature (Fig. 7). Such behaviour suggests that the extended phonons exist in biopolymeric materials, which essentially are expected to be rather disordered. Also glassy materials can display relaxation characteristic of crystalline state, as it was observed for paramagnetic ions in inorganic glasses. For $\mathrm{Y}^{3+}$ ions in phosphate glasses it was found $1 / T_{1}=a T+b T^{9} I_{8}\left(\Theta_{\mathrm{D}} / T\right)$ (Fig. 7) with the second term describing Raman process with a Debye-type phonon spectrum but unusually low Debye temperature $\Theta_{\mathrm{D}}=46 \mathrm{~K}$ [24].

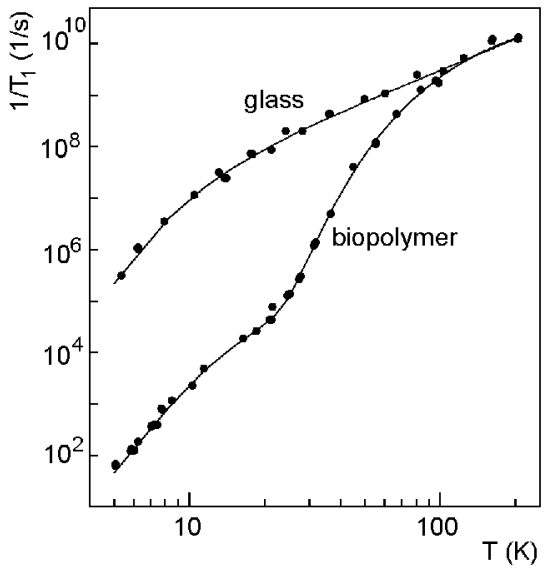

Fig. 7. Temperature dependence of the spin-lattice relaxation rate for $\mathrm{Fe}^{3+}$ in ferrodoxine biopolymer [23], and for $\mathrm{Y}^{3+}$ in phosphate glass [24], showing dominant contributions from ordinary phonon relaxation processes. 


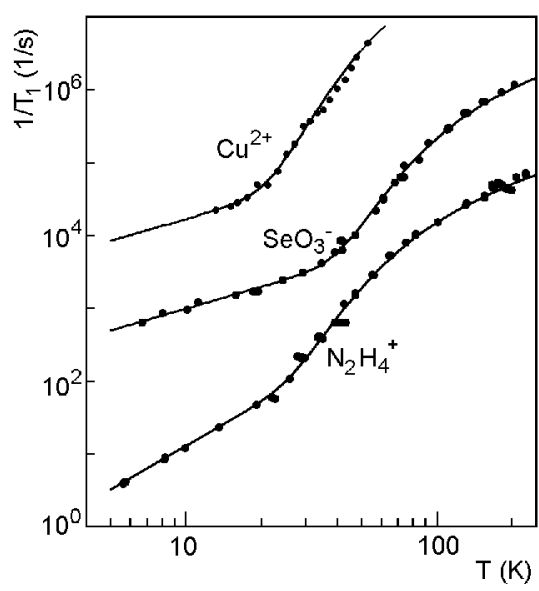

Fig. 8. Comparison of the spin-lattice relaxation rate for $\mathrm{Cu}^{2+}$ and two radicals in single crystals showing amorphous-type effects. For $\mathrm{Cu}^{2+}$ in $\left(\mathrm{NH}_{4}\right)_{2} \mathrm{Mg}\left(\mathrm{SO}_{4}\right)_{2} \cdot 6 \mathrm{H}_{2} \mathrm{O}[26,27]$ relaxation is described by $1 / T_{1}=a T+b T^{9} I_{8}\left(\Theta_{\mathrm{D}} / T\right)$ with a linear term from cross-relaxation via exchange coupled pairs $\left(a=1700 \mathrm{~K}^{-1} \mathrm{~s}^{-1}\right)$ and Raman contribution with $b=0.8 \times 10^{-12} \mathrm{~K}^{-9} \mathrm{~s}^{-1}$ and the Debye temperature $\Theta_{\mathrm{D}}=238 \mathrm{~K}$ (solid line). The relaxation rate for radicals was well fitted to the equation $1 / T_{1}=$ $a T+b T^{2}+c \operatorname{cosech}(\Delta / k T)$ : for $\mathrm{SeO}_{3}$ - radical in $\left(\mathrm{NH}_{4}\right)_{3} \mathrm{H}\left(\mathrm{SeO}_{4}\right)_{2} a=100 \mathrm{~K}^{-1} \mathrm{~s}^{-1}$, $b=0, c=2.34 \times 10^{6} \mathrm{~s}^{-1}$, and $\Delta=216 \mathrm{~cm}^{-1}[27] ;$ for $\mathrm{N}_{2} \mathrm{H}_{4}^{+}$radical in $\mathrm{Li}\left(\mathrm{N}_{2} \mathrm{H}_{5}\right) \mathrm{SO}_{4}$ $a=0, b=0.128 \mathrm{~K}^{-2} \mathrm{~s}^{-1}, c=6.0 \times 10^{4} \mathrm{~s}^{-1}$, and $\Delta=150 \mathrm{~cm}^{-1}[29]$.

Our results and literature data $[13,20,25]$ suggest that the relaxation mechanisms dominating in amorphous materials can dominate also in single crystals when paramagnetic centres are created by doping with paramagnetic ions or by $\gamma$ - or X-ray irradiation. Experimental results indicating such behaviour are shown in Fig. 8. Two effects can appear: linear temperature dependence of the relaxation rate in broad temperature range and ordinary phonon processes do not operate. The first effect appears sometimes in paramagnetic ion doped crystals as a linear temperature dependence of the relaxation rate at low temperatures, as in $\left(\mathrm{NH}_{4}\right)_{2} \mathrm{Mg}\left(\mathrm{SO}_{4}\right)_{2} \cdot 6 \mathrm{H}_{2} \mathrm{O}[26,27]$. The range of temperature and parameters of the dependence vary from crystal to crystal, and depend on the ion concentration and crystal growing conditions. This cannot be explained as due to the direct single-phonon relaxation process since no characteristic quadratic dependence of $1 / T_{1}$ on external magnetic field is observed. This effect results rather from a non-uniform distribution of the doped ions and cross-relaxation via exchange coupled pairs as described by Eq. (6) for $\Delta_{\mathrm{ST}} \ll k T$.

The electron spin-lattice relaxation of free radicals formed by $\gamma$-irradiation in $\left(\mathrm{NH}_{4}\right)_{3} \mathrm{H}\left(\mathrm{SeO}_{4}\right)_{2}[28]$ and $\mathrm{Li}\left(\mathrm{N}_{2} \mathrm{H}_{5}\right) \mathrm{SO}_{4}$ [29] single crystals is described by Eq. (9) without any contribution from the ordinary phonon relaxation processes charac- 
teristic of single crystals. It indicates that even for relatively low irradiation dose order of $1 \mathrm{kGy}$ producing about $10^{18}$ radicals $/ \mathrm{cm}^{3}$ local amorphisation of a crystal appears suppressing phonon dynamics around radiation damage centres. Such behaviour is generally observed for free radicals generated by $\gamma$ rays.

\section{Conclusions}

Electron spin-lattice relaxation in polymers and glassy materials is much slower than that in crystalline solids and it allows, as a rule, observation of the electron spin echo signal and measurements the relaxation rate even at room temperature, whereas for paramagnetic ions in single crystals such measurements can be performed at low temperatures (below $100 \mathrm{~K}$ as a rule) only. Temperature dependence of the relaxation rate $1 / T_{1}$ displays universal type behaviour in amorphous solids with linear increase at low and intermediate temperatures and exponential-type behaviour at higher temperatures.

In some amorphous materials like biopolymers or inorganic glasses the relaxation dominated by extended phonons processes is observed. This relaxation is similar to that in crystals with a Debye-type phonon spectrum but with unusually low Debye temperature $\Theta_{\mathrm{D}}<50 \mathrm{~K}$. On the other hand, single crystals can display the temperature dependence of the spin relaxation characteristic of amorphous materials when doped paramagnetic ions are non-uniformly distributed in the host lattice. Also in single crystals with free radicals generated by $\mathrm{X}$ - or $\gamma$-irradiation local crystal amorphisation by the radiation damage can suppress the ordinary phonon relaxation processes and enhances contributions to $1 / T_{1}$ from cross-relaxation and local dynamic processes. This implies that even in single crystals when one discusses the linewidth of cw-EPR spectra the contributions characteristic of amorphous materials should be taken into account and vice versa, in cw-EPR of amorphous materials the crystalline contributions should be considered.

\section{References}

[1] S.R. Elliott, Physics of Amorphous Materials, Longmans, New York 1990.

[2] Amorphous Solids: Low-Temperature Properties, Ed. W.A. Phillips, Springer, Berlin 1981.

[3] D.A. Parshin, Fiz. Tverd. Tela 36, 1809 (1994).

[4] E. Courtens, M. Foret, B. Hehlen, R. Vacher, Sol. State Commun. 117, 187 (2001).

[5] P.K. Zinsou, D. Vergnoux, G. Ablart, J. Pescia, S.K. Misra, R. Berger, Appl. Magn. Res. 11, 487 (1996).

[6] A. Abragam, B. Bleaney B, Electron Paramagnetic Resonance of Transition Ions, Clarendon Press, Oxford 1970.

[7] S.K. Hoffmann, J. Goslar, Appl. Magn. Res. 14, 293 (1998). 
[8] S.K. Misra, Spectrochim. Acta A 54, 2257 (1998).

[9] W.A. Phillips, J. Low Temp. Phys. 7, 351 (1972).

[10] S. Estalji, O. Kanert, J. Steiner, H. Jain, K.L. Ngai, Phys. Rev. B 43, 7481 (1991).

[11] M.K. Bowman, L. Kevan, J. Phys. Chem. 81, 456 (1977).

[12] J.G. Castle, D.W. Feldman, Phys. Rev. 137, 671 (1965).

[13] L.K. Aminov, I.N. Kurkin, D.A. Lukoyanov, Appl. Magn. Res. 14, 447 (1998).

[14] S.B. Stevens, H.J. Stapleton, Phys. Rev. B 42, 9794 (1990).

[15] M.B. Schultz, C.D. Jeffries, Phys. Rev. 149, 270 (1966).

[16] M.M. Bowman, in: Magnetic Resonance of Carbonaceous Solids, Eds. R.E. Botto, Y. Sanada, American Chemical Society, Washington 1993.

[17] S.K. Hoffmann, W. Hilczer, IEEE Trans. Diel. Electr. Insul. 9, 316 (2002).

[18] S.K. Hoffmann, I. Polus, W. Hilczer, J. Goslar, S. Kiczka, B. Doczekalska, Pol. J. Chem. 77, 87 (2003).

[19] J. Murphy, Phys. Rev. 145, 241 (1966).

[20] L.R. Dalton, A.L. Kwiram, J.A. Cowen, Chem. Phys. Lett. 17, 495 (1972).

[21] J. Pescia, S.K. Misra, M. Zaripov, Phys. Rev. Lett. 83, 1866 (1999).

[22] J. Pescia, S.K. Misra, M. Zaripov, Y. Servant, Phys. Rev. B 59, 9442 (1999).

[23] J.P. Allen, J.T. Colvin, D.G. Stinson, C.P. Flynn, H.J. Stapleton, Biophys. J. 38, 299 (1982).

[24] D. Vergnoux, P.K. Zinsou, M. Zaripov, G. Ablart, J. Pescia, S.K. Misra, R. Rakhmatullin, S. Orlinskii, Appl. Magn. Res. 11, 493 (1996).

[25] J. Goslar, S.K. Hoffmann, W. Hilczer, Sol. State Commun. 121, 423 (2002).

[26] S.K. Hoffmann, M.A. Augustyniak, J. Goslar, W. Hilczer, Mol. Phys. 95, 1265 (1998).

[27] S.K. Hoffmann, J. Goslar, W. Hilczer, M.A. Augustyniak, M. Marciniak, J. Phys. Chem. A 102, 1697 (1998).

[28] W. Hilczer, S.K. Hoffmann, J. Goslar, M. Augustyniak, Sol. State Commun. 85, 585 (1993).

[29] P. Morawski, S.K. Hoffmann, W. Hilczer, J. Goslar, Acta Phys. Pol. A 91, 1121 (1997).

[30] S.K. Hoffmann, W. Hilczer, J. Goslar, M.A. Augustyniak-Jablokow, J. Phys. Condens. Matter 13, 7443 (2001).

[31] L.Y. Dzavakhishvili, G.D. Kethiladze, T.I. Sanadze, Fiz. Tverd. Tela 10, 2957 (1969).

[32] V.I. Volkov, V.I. Muromtsev, K.K. Pukhov, V.G. Cheredintsev, Fiz. Tverd. Tela 19, 1230 (1977). 